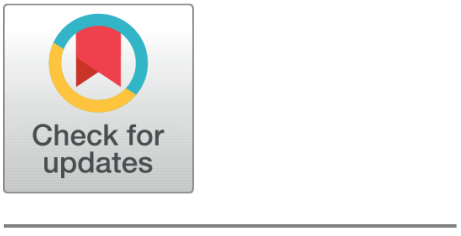

open ACCESS

Received: 03.10.2020

Accepted: 19.03 .2021

Published: 20.04.2021

Citation: Meenapriya P,

Uma Maheswari K,

Nirmala P Ratchagar (2021) A

Two-phase mixture model of

Atmospheric Aerosols in the channel

with an applied electric and

magnetic field. Indian Journal of

Science and Technology 14(13):

1056-1070. https://doi.org/

10.17485/IJST/v14i13.1796

* Corresponding author.

umamohansusd@gmail.com

Funding: None

Competing Interests: None

Copyright: () 2021 Meenapriya et al. This is an open access article distributed under the terms of the Creative Commons Attribution License, which permits unrestricted use, distribution, and reproduction in any medium, provided the original author and source are credited.

Published By Indian Society for Education and Environment (iSee)

ISSN

Print: 0974-6846

Electronic: 0974-5645

\section{A Two-phase mixture model of Atmospheric Aerosols in the channel with an applied electric and magnetic field}

\author{
P Meenapriya ${ }^{1}$, K Uma Maheswari ${ }^{1}{ }^{*}$, Nirmala P Ratchagar ${ }^{1}$ \\ 1 Department of Mathematics, Annamalai University, Chidambaram, 608 002, India
}

\section{Abstract}

Objectives: A mathematical model is constructed to investigate the concentration of aerosol mixture in the atmosphere. Aerosol is the significant constituent of the atmosphere, as it is playing a key role in environmental effects including air quality degradation, smoke-fog related accidents, acid rain formation, and visibility issues. The objective of this paper is to formulate the effects of aerosol mixture, and it is monitored through its concentration in the presence and absence of chemical reaction. The effects of concentration of aerosol mixture has to be reduced so as to enhance visibility. Methodology: A two-dimensional schematic geometry is considered which comprises the channel extended to infinity in the $x$-axis bounded by porous layers, also electric and magnetic field are applied transversely. A general exact solution of the dimensionless governing partial differential equations are obtained using perturbation technique with appropriate boundary conditions. Findings: Detailed results of velocity and concentration of the aerosol mixture were presented graphically. The graphical representation validated the accuracy of attained results with an encouraging level of agreement. As a measure to enhance the effects of air visibility, significant conclusions are specified using the obtained results.

Keywords: atmospheric fluid; coagulation; concentration; porous layers; mixture theory

\section{Introduction}

Atmospheric aerosols are extremely small, finely distributed, liquid or solid particles suspended in a gaseous medium. Both natural and anthropogenic aerosol particles have been playing a major role in the global climate for several years. Due to coagulation, aerosols bind each other and deforms in the atmosphere resulting in either larger or smaller particles than its original size and shape. The particles that are formed due to coagulation, have a direct impact on fog formation, cloud physics, reduction of visibility, etc. Thus, there is a requirement of evaluating the concentration of the mixture of aerosols and atmospheric fluid using mixture theory.

A handful of research work was attempted in the field of mixture theory. Barry et al ${ }^{(1)}$ developed the governing equations for flow in porous layers and obtained solutions 
using mixture theory. Bowen ${ }^{(2)}$ established the use of thermodynamics of mixture to porous media for an incompressible fluid. Labonokitov et $\mathrm{al}^{(3)}$ used Cauchy-Born hypothesis to show that there is an interaction force between solid and fluid and provided linear boundary conditions to check the consistency of porosity theory.

Many studies were conducted in the coagulation of aerosols in particular. Anand et al ${ }^{(4)}$ bring forth a numerical study of the coagulation of aerosols and its number concentration in air. The concentration and source strength of vapors has been measured using aerosol condensation analytically by Dal Maso et al ${ }^{(5)}$. Gan Fu Zan et al ${ }^{(6)}$ elucidated the role of the fluid shear rate on the particle coagulation using LES technique and Taylor- series expansion moment method. The mathematical model for concentration of pollution is developed based on the coagulation and deposition process by Keller and Siegmann ${ }^{(7)}$. The results of both theoretical and experimental analysis of nanoparticle aerosols in the atmosphere were studied by Seipenbusch et $\mathrm{al}^{(8)}$.

Different analytical approaches are existing to solve the derived partial differential equations. Dettman ${ }^{(9)}$ exhibited a perturbation technique in elliptic and hyperbolic problems and showed how the method tends to unify the behavior of variety of problems in perturbation theory. Rudraiah et al ${ }^{(10)}$ solved the problem of dispersion of aerosol mixture using the perturbation technique.

Meenapriya and Ratchagar ${ }^{(11)}$ attempted to study coagulation of aerosol in couple stress fluid using generalised dispersion method. The concentration and dispersion of air pollutants was evaluated in an applied electric and magnetic field by Meenapriya et $\mathrm{al}^{(12,13)}$. Dhange and Sankad ${ }^{(14)}$ investigated the dispersion and chemical effects of the sinusoidal stream of an MHD couple stress fluid analytically. Varaksin ${ }^{(15)}$ reviewed the current state of aerosol process modeling which pertains to gasaerosol partitioning, and highlighted the gap between current understanding of gas-aerosol partitioning from measurements, comprehensive box models and parameterizations for large-scale modelling.

These studies urge to study about aerosol mixture in a channel bounded by porous beds with the externally applied electric and magnetic fields. The concentration of the aerosol mixture is evaluated both in the presence and absence of homogeneous first-order chemical reaction. Numerical calculations are computed using MATHEMATICA and the results are portrayed graphically by varying parameters like Hartmann number, electric number, reaction rate parameter, porous parameter. The potrayed graphical results helps to analyze the effects of air visibility through the concentration of aerosol mixture.

\section{Mathematical Formulation}

A two-dimensional turbulent flow of chemically reactive aerosols in a poorly conducting atmospheric fluid bounded by porous layers is modelled as shown in Figure 1 . Assuming at each point in the channel is simultaneously occupied by both aerosols and atmospheric fluid. The component is represented by $\beta$, where $\beta=s$ for solid phase (aerosols) and $\beta=f$ for fluid phase (atmospheric fluid). Both solid and fluid are assumed to be intrinsically incompressible.

Uniform magnetic field of strength $B_{0}$ and electric field through electrodes with electric potentials $\varphi=(V h x) a t y=$ $-h, \varphi=\left(V h\left(x-x_{0}\right)\right)$ at $y=h$ are applied transversely through porous boundaries. The channel is symmetric about $x$-axis which extends to infinity in both directions with applied pressure gradient $\frac{\partial p}{\partial t}=G$. The porous medium considered here is homogenous and isotropic. The concentration of aerosols with and without chemical reaction both in the solid phase and fluid phase are calculated respectively.

Basic governing equations are based on poro-elasticity and are derived using mixture theory and Reynolds averaging procedure.

Equation of continuity

$$
\nabla \cdot\left(\varphi^{\beta} q_{i}^{\beta}\right)=0
$$

Equation of Momentum

$$
\rho^{\beta}\left[\frac{\partial q_{i}^{\beta}}{\partial t}+q_{j}^{\beta} \frac{\partial q_{i}^{\beta}}{\partial x_{j}}\right]=-\varphi^{\beta} \frac{\partial p}{\partial x_{i}}+\mu_{\beta}\left[\frac{\partial^{2} q_{i}^{\beta}}{\partial x^{2}}+\frac{\partial^{2} q_{i}^{\beta}}{\partial y^{2}}\right] \pm K\left(q_{i}^{s}-q_{i}^{f}\right)
$$

Where $q_{i}^{\beta}$ is the velocity of aerosols (if $\beta=s$ ) and $q_{i}^{\beta}$ is the velocity of the atmospheric fluid (if $\beta=f$ ) with $i=1,2$ and $j=1,2$. Also $p$ is the pressure of the fluid, $\mathrm{K}$ is the linear drag coefficient that is, Darcy resistance offered by solid to fluid. The term $\frac{\partial p}{\partial x_{i}}$ is the pressure gradient, $\varphi^{s}$ is the aerosol volume fraction and $\varphi^{f}$ is the fluid volume fraction, $\rho^{\beta}$ is the density of the mixture and $\mu_{\beta}$ is the viscosity of the mixture. 


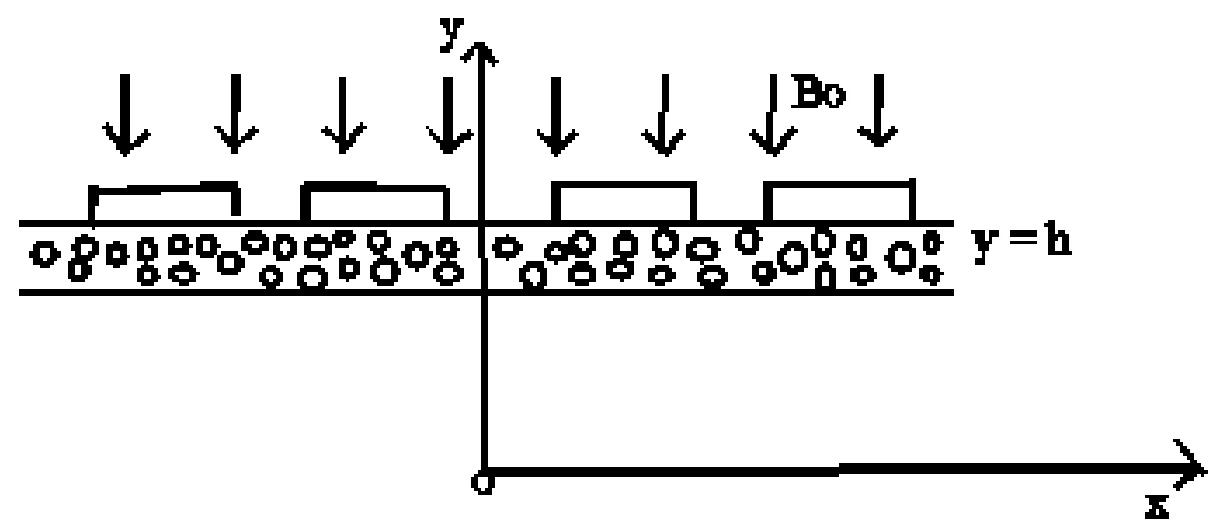

Atmospheric Fhuid

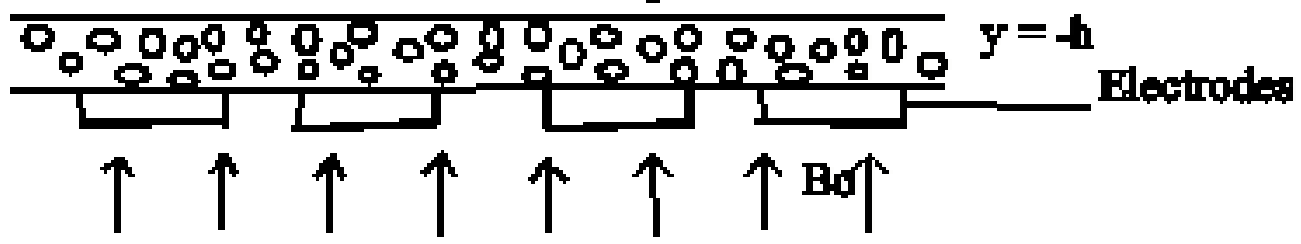

Fig 1. Physical Configuration

Equation of Species

$$
\left(\frac{\partial C_{i}}{\partial t}+u^{\beta} \frac{\partial C_{i}}{\partial x}+v^{\beta} \frac{\partial C_{i}}{\partial y}\right)=D_{e}\left(\frac{\partial^{2} C_{i}}{\partial x^{2}}+\frac{\partial^{2} C_{i}}{\partial y^{2}}\right)-R C_{i}
$$

For $i=1,2$ let $C_{1}$ denotes mean aerosol concentration with chemical reaction, $C_{2}$ signifies mean aerosol concentration without chemical reaction, $D_{e}$ is eddy diffusion coefficient and $\mathrm{R}$ represents first-order chemical reaction.

To derive a cartesian form of governing equations let the components of velocity be $q_{1}^{s}=u^{s}$, is the $x$-component of aerosol, $q_{1}^{f}=u^{f}$, is the $x$-component of the atmospheric fluid, $q_{2}^{s}=v^{s}$, is the $y$-component of aerosol, $q_{2}^{f}=v^{f}$, is $y$-component of atmospheric fluid. Let the components of $x_{i}$ are $x_{1}=x, x_{2}=y$.

The Cartesian form of equations (1) to (3) are,

Continuity equation

$$
\frac{\partial}{\partial x}\left(\varphi v^{s}+\varphi^{f} v^{f}\right)+\frac{\partial}{\partial y}\left(\varphi^{s} v^{s}+\varphi^{f} v^{f}\right)=0
$$

$\mathrm{x}$-momentum equation

$$
\begin{aligned}
\rho^{\beta} & {\left[\frac{\partial u^{\beta}}{\partial t}+u^{\beta} \frac{\partial u^{\beta}}{\partial x}+v^{\beta} \frac{\partial u^{\beta}}{\partial y}\right]=-\varphi^{\beta} \frac{\partial p}{\partial x}+\mu_{\beta}\left(\frac{\partial^{2} u^{\beta}}{\partial x^{2}}+\frac{\partial^{2} u^{\beta}}{\partial y^{2}}\right) } \\
& -K\left(u^{s}-u^{f}\right)+\rho_{e} E_{x}-\sigma_{0} B_{0}^{2} u^{\beta}
\end{aligned}
$$

$\mathrm{y}$-momentum equation

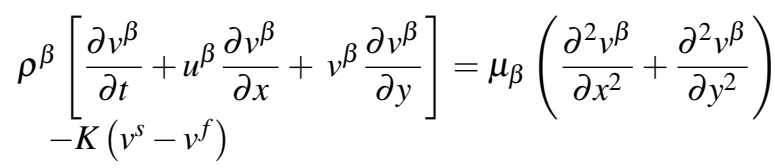

Species equation

$$
\left(\frac{\partial C_{i}}{\partial t}+u^{\beta} \frac{\partial C_{i}}{\partial x}+v^{\beta} \frac{\partial C_{i}}{\partial y}\right)=D\left(\frac{\partial^{2} C_{i}}{\partial x^{2}}+\frac{\partial^{2} C_{i}}{\partial y^{2}}\right)-R C_{i}
$$


For $D_{e}=D+K_{x x}=D+K_{y y}$, where the eddy diffusivity coefficients $K_{y y}, K_{y y}$ are assumed to be small, hence eddy diffusion coefficient $\left(D_{e}\right)$ is molecular diffusion coefficient $(D)$. To solve the governing equations Beaver Joseph slip boundary conditions $^{(16)}$ are used to study the boundary effects of velocity.

Boundary conditions for velocity are

$$
\begin{gathered}
u^{\beta}=0, v^{\beta}=\frac{h^{2} G_{0}}{\mu_{\beta}} \varepsilon e^{i(\alpha x+w t)}, \text { at } y=0 \\
\frac{\partial u^{\beta}}{\partial y}=\frac{-\alpha_{p}}{\sqrt{k}}\left(u^{\beta}-u_{p}\right), v^{\beta}=\frac{h^{2} G_{0}}{\mu_{\beta}}\left(1+\varepsilon e^{i(\alpha x+w t)}\right), \text { at } y=h \\
\frac{\partial u^{\beta}}{\partial y}=\frac{\alpha_{p}}{\sqrt{k}}\left(u^{\beta}-u_{p}\right), \text { at } y=-h
\end{gathered}
$$

For Concentration

$$
\begin{gathered}
C_{i}=C_{0} \varepsilon e^{i(\alpha x+w t)}, \text { at } y=0 \\
C_{i}=C_{0}\left(1+\varepsilon e^{i(\alpha x+w t)}\right) \text {, at } y=h \\
C_{i}=C_{0}, \text { at } y=-h
\end{gathered}
$$

Where $u_{p}$ is the Darcy velocity of the porous layer, $\alpha_{p}$ is the slip parameter, $k$ is the permeability of the porous layer, $\alpha$ is the streamwise wave number, $\mathrm{w}$ is the frequency parameter, $\varepsilon$ is the perturbation parameter, $\mathbf{i}$ is the imaginary number, $C_{0}$ is the dimensionless concentration and $u_{p}=-\frac{k}{\mu} \frac{\partial p}{\partial x}$ represents Darcy law. To make (4) to (13) non-dimension the following dimensionless quantities are introduced.

$$
\begin{aligned}
& x^{*}=\frac{x}{h} ; y^{*}=\frac{y}{h} ; t^{*}=\frac{t}{t_{0}} ; u^{s *}=\frac{\mu_{s} u^{s}}{h^{2} G_{0}} ; u^{f *}=\frac{\mu_{f} u^{f}}{h^{2} G_{0}} ; G^{*}=\frac{G}{G_{0}} ; v^{s *}=\frac{\mu_{s} v^{s}}{h^{2} G_{0}} ; \\
& v^{f *}=\frac{\mu_{f} v^{f}}{h^{2} G_{0}} ; \rho_{e}^{*}=\frac{\rho_{e}}{\left(\varepsilon_{0} \frac{V}{h^{2}}\right)} ; p^{*}=\frac{p}{\rho\left(\frac{V}{h}\right)^{2}} ; u_{p}^{*}=\frac{u_{p}}{\left(\frac{V}{h}\right)} ; E_{x}^{*}=\frac{E_{x}}{\left(\frac{V}{h}\right)} ; \\
& \varphi^{f *}=\frac{\varphi^{f}}{V} ; \varphi^{s *}=\frac{\varphi^{s}}{V} ; \beta^{2}=\frac{h^{2} R}{D} ; C_{i}^{*}=\frac{C_{i}}{C_{0}} ;
\end{aligned}
$$

The reaction rate parameter is $\beta$ and $V$ is the applied constant electric potential due to embedded electrodes at the boundaries. The porous parameter $\sigma=\frac{h}{\sqrt{k}}$, the electric number $W_{e}=\frac{\varepsilon_{0} V^{2}}{\mu_{\beta}}$, Hartmann number is $M^{2}=\frac{\sigma_{0} B_{0}{ }^{2} h^{2}}{\mu}$. After dimensionalising the above governing equations and boundary conditions neglecting asterisk we obtain,

$$
\frac{\varphi^{f}}{\mu_{f}}\left(\frac{\partial u^{f}}{\partial x}+\frac{\partial v^{f}}{\partial y}\right)+\frac{\varphi^{s}}{\mu_{s}}\left(\frac{\partial u^{s}}{\partial x}+\frac{\partial v^{s}}{\partial y}\right)=0
$$

x-momentum solid phase

$$
\frac{\partial^{2} u^{s}}{\partial x^{2}}+\frac{\partial^{2} u^{s}}{\partial y^{2}}-A_{1} \frac{\partial u^{s}}{\partial t}-A_{2}\left[u^{s} \frac{\partial u^{s}}{\partial x}+v^{s} \frac{\partial u^{s}}{\partial y}\right]-\varphi^{s} A_{3} \frac{\partial p}{\partial x}-A_{4}\left(u^{s}-u^{f} r_{3}\right)+A_{5} W e\left(\rho_{e} E_{x}\right)-M^{2} u^{s}=0
$$

$\mathrm{X}$ - momentum fluid phase

$$
\frac{\partial^{2} u^{f}}{\partial x^{2}}+\frac{\partial^{2} u^{f}}{\partial y^{2}}-A_{6} \frac{\partial u^{f}}{\partial t}-A_{7}\left[u^{f} \frac{\partial u^{f}}{\partial x}+v^{f} \frac{\partial u^{f}}{\partial y}\right]-\varphi^{f} A_{3} \frac{\partial p}{\partial x}+A_{4}\left(u^{s}-r_{3} u^{f}\right)+A_{5} W e\left(\rho_{e} E_{x}\right)-M^{2} u^{f}=0
$$


y- momentum solid phase

$$
\frac{\partial^{2} v^{s}}{\partial x^{2}}+\frac{\partial^{2} v^{s}}{\partial y^{2}}-A_{1} \frac{\partial v^{s}}{\partial t}-A_{2}\left[u^{s} \frac{\partial v^{s}}{\partial x}+v^{s} \frac{\partial v^{s}}{\partial y}\right]-A_{4}\left(v^{s}-v^{f} r_{3}\right)=0
$$

y- momentum fluid phase

$$
\frac{\partial^{2} v^{f}}{\partial x^{2}}+\frac{\partial^{2} v^{f}}{\partial y^{2}}-A_{6} \frac{\partial v^{f}}{\partial t}-A_{7}\left[u^{f} \frac{\partial v^{f}}{\partial x}+v^{f} \frac{\partial v^{f}}{\partial y}\right]+A_{4}\left(v^{s}-v^{f} r_{3}\right)=0
$$

\section{Concentration}

$$
\frac{\partial^{2} C_{i}}{\partial x^{2}}+\frac{\partial^{2} C_{i}}{\partial y^{2}}-c_{1}\left(\frac{\partial C_{i}}{\partial t}\right)-c_{2}\left(u^{\beta} \frac{\partial C_{i}}{\partial x}+v^{\beta} \frac{\partial C_{i}}{\partial y}\right)-\beta C_{i}=0
$$

The dimensionless boundary conditions for velocity are given by,

$$
\begin{gathered}
u^{\beta}=0, v^{\beta}=\varepsilon e^{i(\alpha x+w t)}, \text { at } y=0 \\
\frac{\partial u^{\beta}}{\partial y}=-\alpha \sigma\left(u^{\beta}-u_{p}\right), v^{\beta}=\left(1+\varepsilon e^{i(\alpha x+w t)}\right) \text {, at } y=1 \\
\frac{\partial u^{\beta}}{\partial y}=\alpha \sigma\left(u^{\beta}-u_{p}\right), \text { at } y=-1
\end{gathered}
$$

For Concentration

$$
\begin{gathered}
C_{i}=\varepsilon e^{i(\alpha x+w t)}, \text { at } y=0 \\
C_{i}=\left(1+\varepsilon e^{i(\alpha x+w t)}\right), \text { at } y=1 \\
C_{i}=1, \text { at } y=-1
\end{gathered}
$$

To determine the velocity of the fluid, we need to evaluate the value of $\rho_{e} E_{x}$ in x-momentum equations. Using conservation of charges equation and Maxwell's equation, the value of $\rho_{e} E_{x}$ is calculated as given below.

Conservation of charges

$$
\frac{\partial \rho_{e}}{\partial t}+(q . \nabla) \rho_{e}+\nabla . J=0
$$

Maxwell's equation

$$
\begin{gathered}
\nabla . E=\frac{\rho_{e}}{\epsilon_{0}}(\text { Gauss law }) \\
-\frac{\partial B_{0}}{\partial t}=\nabla \times E \quad(\text { Faraday's law }) \\
J=\sigma_{c}\left(E+u \times B_{0}\right)\left(\text { Ohm }^{\prime} \text { s law }\right)
\end{gathered}
$$


In a poorly conducting fluid, the induced magnetic field is negligibly small compared to an electric field and hence the current density is $J=\sigma_{c} E$ and the electrical conductivity $\sigma_{c} \ll 1$ and hence any perturbation on it is negligible and so it depends upon the conduction temperature $T_{b}$.

So (26) reduces to $\frac{\partial J}{\partial y}=0$ and the equation of electric potential $\varphi$ reduces to,

$$
\begin{gathered}
\frac{\partial^{2} \varphi}{\partial y^{2}}+\frac{1}{\sigma_{c}} \frac{\partial \varphi}{\partial y} \frac{\partial \sigma_{c}}{\partial y}=0 \\
\text { with } \sigma_{c}=\sigma_{0}\left(1+\alpha_{h}\left(T_{b}-T_{0}\right)\right)
\end{gathered}
$$

Where $\alpha_{h}$ is the volumetric expansion coefficient and $T_{b}$ is the solution of equation $\frac{d^{2} T_{b}}{d y^{2}}=0$ which is solved using dimensionless quantities, $T_{b}{ }^{*}=\frac{T_{b}}{\triangle T} ; \eta=\frac{y}{h}$; and the boundary conditions are $T_{b}=T_{0}$ at $\eta=-1$, and $T_{b}=T_{1}$ at $\eta=1$. Hence we obtain the equation $T_{b}-T_{0}=\frac{\Delta T(y+h)}{2 h}$, where $\triangle T=T_{1}-T_{0}$.

Using this value in (31), we get $\sigma_{c}=\sigma_{0} e^{\alpha_{c}(y+h)}$ and solving (30) using the boundary condition, $\varphi=x$ Pe at $y=-1$, and $\varphi=$ $\left(x-x_{0}\right)$ Pe at $y=1$, we get the value $\varphi=\left(x-\frac{x_{0} e^{\alpha_{c} h}-e^{-\alpha_{c} y}}{2 \sinh \alpha_{c}}\right) P e$

Now consider, $\rho_{e} E_{x}=(\nabla . E) E_{x}$ (from (27)

Since $E_{x}=-1$, and $E=-\nabla \varphi$ then $\rho_{e} E_{x}=-\nabla^{2} \varphi(-1)$

Hence $\rho_{e} E_{x}=\frac{\alpha_{c}^{2} x_{0} e^{-\alpha_{c} y}}{2 \sinh \alpha_{c}}=a_{2} e^{-\alpha_{c} y P e}$, where $a_{2}=\frac{\alpha_{c}^{2} x_{0}}{2 \sinh \alpha_{c}}$

After substituting $\rho_{e} E_{x}$, equations (15) and (16) becomes

$$
\begin{gathered}
\frac{\partial^{2} u^{s}}{\partial x^{2}}+\frac{\partial^{2} u^{s}}{\partial y^{2}}-A_{1} \frac{\partial u^{s}}{\partial t}-A_{2}\left[u^{s} \frac{\partial u^{s}}{\partial x}+v^{s} \frac{\partial u^{s}}{\partial y}\right]-\varphi^{s} A_{3} \frac{\partial p}{\partial x}-A_{4}\left(u^{s}-u^{f} r_{3}\right)+A_{5} W e\left(a_{2} e^{-\alpha_{c} y}\right)-M^{2} u^{s}=0 \\
\frac{\partial^{2} u^{f}}{\partial x^{2}}+\frac{\partial^{2} u^{f}}{\partial y^{2}}-A_{6} \frac{\partial u^{f}}{\partial t}-A_{7}\left[u^{f} \frac{\partial u^{f}}{\partial x}+v^{f} \frac{\partial u^{f}}{\partial y}\right]-\varphi^{f} A_{3} \frac{\partial p}{\partial x}+A_{4}\left(u^{s}-r_{3} u^{f}\right)+A_{5} W e\left(a_{2} e^{-\alpha_{c} y}\right)-M^{2} u^{f}=0
\end{gathered}
$$

\subsection{Method of Solution}

To evaluate the values of velocity and concentration of the aerosol mixture, Perturbation technique is used. Disintegrating the flow variables into steady base state quantities denoted by upper case and two-dimensional linear perturbed quantities denoted by tilde $(\sim)$ symbol as follows,

$$
\begin{gathered}
u(x, y)=U_{B}(y)+\widetilde{u}(y) \varepsilon e^{i(\alpha x+\omega t)}+O\left(\varepsilon^{2}\right) \\
v(x, y)=\widetilde{v}(y) \varepsilon e^{i(\alpha x+\omega t)}+O\left(\varepsilon^{2}\right) \\
p(x, y)=p_{B}(y)+\widetilde{p}(y) \varepsilon e^{i(\alpha x+\omega t)}+O\left(\varepsilon^{2}\right) \\
C_{i}(x, y)=C_{B i}(y)+\widetilde{C}_{i}(y) \varepsilon e^{i(\alpha x+\omega t)}+O\left(\varepsilon^{2}\right)
\end{gathered}
$$

After decomposing the above equation into base and perturbed parts, the solution of the base part are obtained analytically and that of the perturbed part are obtained numerically. Let the perturbation parameter $\varepsilon=A_{4} r_{3}=\frac{\mu_{s}}{\mu_{f}} \frac{k h^{2}}{\mu_{s}}$ is assumed to be so small, where $A_{4} \ll r_{3}$. Assuming the flow to be steady, and splitting (14), (17) to (25), (32), (33) and neglecting the higher orders of perturbation parameter $\varepsilon$, we obtain the following set of partial differential equations. 
Base part equations

$$
\begin{gathered}
\frac{\partial^{2} U_{B}^{s}}{\partial y^{2}}-\varphi^{s} A_{3} \frac{\partial p_{B}}{\partial x}+A_{5} W e a_{2} e^{-\alpha_{c} y}-M^{2} U_{B}^{s}=0 \\
\frac{\partial^{2} U_{B}^{f}}{\partial y^{2}}-\varphi^{f} A_{3} \frac{\partial p_{B}}{\partial x}+A_{5} W e a_{2} e^{-\alpha_{c} y}-M^{2} U_{B}^{f}=0 \\
\frac{\partial^{2} C_{B_{i}}}{\partial y^{2}}-\beta C_{B_{i}}=0
\end{gathered}
$$

Perturbed part equations

$$
\begin{gathered}
\left(\frac{\partial \widetilde{v^{f}}}{\partial y}-a_{3} \widetilde{u^{f}}\right) r_{1}+\left(\frac{\partial \widetilde{v^{s}}}{\partial y}-a_{3} \widetilde{u^{s}}\right) r_{2}=0 \\
\frac{\partial^{2} \widetilde{u^{s}}}{\partial y^{2}}+\widetilde{u^{s}}\left(A_{1} a_{4}+A_{2} U_{B}^{s} a_{3}-M^{2}-\alpha^{2}\right)-A_{2} \frac{\partial U_{B}^{s}}{\partial y} \widetilde{v}^{s}+\left(A_{3} \varphi^{s} a_{3} \widetilde{p}\right)=0 \\
\frac{\partial^{2} \widetilde{u^{f}}}{\partial y^{2}}+\widetilde{u^{f}}\left(A_{6} a_{4}+A_{7} U_{B}^{f} a_{3}-M^{2}-\alpha^{2}\right)-A_{7} \frac{\partial U_{B}^{f}}{\partial y} \widetilde{v}^{f}+\left(A_{3} \varphi^{f} a_{3} \widetilde{p}\right)=0 \\
\frac{\partial^{2} \widetilde{v^{s}}}{\partial y^{2}}+\widetilde{v^{s}}\left(A_{1} a_{4}+A_{2} U_{B}^{s} a_{3}-\alpha^{2}\right)=0 \\
\frac{\partial^{2} \widetilde{v^{f}}}{\partial y^{2}}+\widetilde{v^{f}}\left(A_{6} a_{4}+A_{7} U_{B}^{f} a_{3}-\alpha^{2}\right)=0 \\
\frac{\partial^{2} \widetilde{C}_{i}}{\partial y^{2}}+\widetilde{C_{i}}\left(c_{2} a_{3} U_{B}^{\beta}+c_{1} a_{4}-\alpha^{2}-\beta\right)-c_{2} \frac{\partial C_{B i}}{\partial y} \widetilde{v}^{\beta}=0
\end{gathered}
$$

where $a_{3}=\alpha \tan (\alpha x+\omega t)$, and $a_{4}=w \tan (\alpha x+\omega t)$,

The base part boundary conditions are

$$
\begin{gathered}
U_{B}^{\beta}=0, C_{B i}=0, \text { at } y=0 \\
C_{B i}=1, \text { at } y=1 \\
\frac{\partial U_{B}^{\beta}}{\partial y}=-\alpha \sigma\left(U_{B}^{\beta}-U_{p_{B}}^{\beta}\right), \text { at } y=1 \\
\frac{\partial U_{B}^{\beta}}{\partial y}=\alpha \sigma\left(U_{B}^{\beta}-U_{p_{B}}^{\beta}\right), C_{B i}=1, \text { at } y=-1
\end{gathered}
$$


and perturbed part boundary conditions are,

$$
\begin{gathered}
\widetilde{u^{\beta}}=0, \widetilde{v^{\beta}}=1, \widetilde{C}_{i}=1 \text {, at } y=0 \\
\widetilde{v^{\beta}}=1, \widetilde{C}_{i}=1 \text {, at } y=1 \\
\frac{\partial \widetilde{u^{\beta}}}{\partial y}=-\alpha \sigma\left(\widetilde{u^{\beta}}-\widetilde{u_{p} \beta}\right) \text {, at } y=1 \\
\widetilde{\partial u^{\beta}} \widetilde{\partial y}=\alpha \sigma\left(\widetilde{u^{\beta}}-\widetilde{u_{p}^{\beta}}\right), \widetilde{C}_{i}=0, \text { at } y=-1
\end{gathered}
$$

Base part solutions

For equations (34) and (35) the solution is

$$
\begin{aligned}
& U_{B}^{s}=A \cosh [M y]+B \sinh [M y]-\frac{A_{9}}{M^{2}}+\frac{A_{8}}{\alpha_{c}{ }^{2}-M^{2}} e^{-\alpha_{c} y} \\
& U_{B}^{f}=C \cosh [M y]+D \sinh [M y]-\frac{A_{9}}{M^{2}}+\frac{A_{8}}{\alpha_{c}{ }^{2}-M^{2}} e^{-\alpha_{c} y}
\end{aligned}
$$

Also the derivatives of $U_{B}^{s}, U_{B}^{f}$ are

$$
\begin{gathered}
\frac{\partial U_{B}^{s}}{\partial y}=A M \sinh [M y]+B M \cosh [M y]-\alpha_{c} \frac{A_{8}}{\alpha_{c}^{2}-M^{2}} e^{-\alpha_{c} y} \\
\frac{\partial U_{B}^{f}}{\partial y}=C M \sinh [M y]+D M \cosh [M y]-\alpha_{c} \frac{A_{8}}{\alpha_{c}^{2}-M^{2}} e^{-\alpha_{c} y}
\end{gathered}
$$

The values of integration constants A,B,C,D are calculated using (43) to (46). The perturbed part equations (38) to (41) are simplified using (51) to (54) as given below,

$$
\begin{gathered}
\frac{\partial^{2} \widetilde{u^{s}}}{\partial y^{2}}+\widetilde{u^{s}}\left(s_{1}-M^{2}+s_{2} \cosh [M y]+s_{3} \sinh [M y]-s_{4} e^{-\alpha_{c} y}\right)-\widetilde{v}^{s}\left(s_{5} \sinh [M y]+s_{6} \cosh [M y]-s_{4} e^{-\alpha_{c} y}\right)+A_{11}=0 \\
\frac{\partial^{2} \widetilde{u^{f}}}{\partial y^{2}}+\widetilde{u^{f}}\left(s_{8}-M^{2}+s_{9} \cosh [M y]+s_{10} \sinh [M y]-s_{11} e^{-\alpha_{c} y}\right)-\widetilde{v^{f}}\left(s_{12} \sinh [M y]+s_{13} \cosh [M y]-s_{14} e^{-\alpha_{c} y}\right)+A_{13}=0 \\
\frac{\partial^{2} \widetilde{v^{s}}}{\partial y^{2}}+\widetilde{v^{s}}\left(s_{15}+s_{2} \cosh [M y]+s_{3} \sinh [M y]+s_{4} e^{-\alpha_{c} y}\right)=0 \\
\frac{\partial^{2} \widetilde{v^{f}}}{\partial y^{2}}+\widetilde{v^{f}}\left(s_{16}+s_{9} \cosh [M y]+s_{10} \sinh [M y]-s_{11} e^{-\alpha_{c} y}\right)=0
\end{gathered}
$$

Where $s_{1}, s_{2}, s_{3}, \ldots \ldots \ldots s_{14}, s_{15}$ are constants obtained when simplification.

The above four perturbed equations (55) to (58) are solved for $\widetilde{u^{s}}, \widetilde{u^{f}}, \widetilde{v^{s}}, \widetilde{v^{f}}$ numerically using MATHEMATICA, and hence velocities for both solid and fluid phases are plotted as graphs respectively. 


\subsubsection{Case 1: Concentration of $\operatorname{smog}\left(C_{1}\right)($ when $\beta \neq \mathbf{0})$}

The base part of $C_{1}$ is $C_{B 1}$ and its solution is obtained from (36) is given by,

$$
C_{B 1}=E \cosh [\sqrt{\beta} y]+F \sinh [\sqrt{\beta} y]
$$

Using boundary conditions (43) to (46) the values $E=0, F=\frac{1}{\sinh [\sqrt{\beta}]}$ are obtained. so,

$$
\begin{gathered}
C_{B 1}=\frac{\sinh \sqrt{\beta} y}{\sinh \sqrt{\beta}} \\
\frac{\partial C_{B 1}}{\partial y}=\frac{\sqrt{\beta} \cosh \sqrt{\beta} y}{\sinh \sqrt{\beta}}
\end{gathered}
$$

For solid phase:

Equation (42) becomes,

$$
\frac{\partial^{2} \widetilde{C}_{1}}{\partial y^{2}}+\widetilde{C_{1}}\left(c_{2} a_{3} U_{B}^{s}+c_{1} a_{4}-\alpha^{2}-\beta\right)-c_{2} \frac{\partial C_{B 1}}{\partial y} \widetilde{v}^{s}=0
$$

Using (51) and (60), the above equation becomes,

$$
\frac{\partial^{2} \widetilde{C}_{1}}{\partial y^{2}}+\widetilde{C_{1}}\left(A_{15}+s_{17} \cosh [M y]+s_{18} \sinh [M y]-s_{19}+s_{20} e^{-\alpha_{c} y}\right)-A_{16} \cosh [\sqrt{\beta} y] \widetilde{v}^{s}=0
$$

For fluid phase:

Equation (42) becomes,

$$
\frac{\partial^{2} \widetilde{C}_{1}}{\partial y^{2}}+\widetilde{C_{1}}\left(c_{2} a_{3} U_{B}^{f}+c_{1} a_{4}-\alpha^{2}-\beta\right)-c_{2} \frac{\partial C_{B 1}}{\partial y} \widetilde{v}^{f}=0
$$

Using (52) and (60), the above equation becomes,

$$
\frac{\partial^{2} \widetilde{C}_{1}}{\partial y^{2}}+\widetilde{C_{1}}\left(A_{15}+s_{17} \cosh [M y]+s_{18} \sinh [M y]-s_{19}+s_{20} e^{-\alpha_{c} y}\right)-A_{17} \cosh [\sqrt{\beta} y] \widetilde{v}^{f}=0
$$

These perturbed equations (63), (65) are solved numerically subject to the boundary conditions prescribed in (47) to (50). Graphs are plotted for concentration with chemical reaction $\left(C_{1}\right)$ for both solid and fluid phase using MATHEMATICA.

\subsubsection{Case 2: Concentration of Haze $C_{2}$ (when $\beta=\mathbf{0}$ )}

The base part of $C_{2}$ is $C_{B 2}$ whose equation from (36) is

$$
\frac{\partial^{2} C_{B_{2}}}{\partial y^{2}}=0
$$

and its solution is given by,

$$
C_{B 2}=G y+H
$$

Using boundary conditions (43) to (46) we get, $G=1, H=0$. so,

$$
C_{B 2}=y
$$

$$
\frac{\partial C_{B 2}}{\partial y}=1
$$


For solid phase:

Equation (42) becomes,

$$
\frac{\partial^{2} \widetilde{C}_{2}}{\partial y^{2}}+\widetilde{C_{2}}\left(c_{3} a_{3} U_{B}^{s}+c_{1} a_{4}-\alpha^{2}\right)-c_{3} \frac{\partial C_{B 2}}{\partial y} \widetilde{v}^{s}=0
$$

Using (51) and (69), the above equation becomes,

$$
\frac{\partial^{2} \widetilde{C}_{2}}{\partial y^{2}}+\widetilde{C_{2}}\left(A_{18}+s_{17} \cosh [M y]+s_{18} \sinh [M y]+s_{19}+s_{20} e^{-\alpha_{c} y}\right)-c_{2} \widetilde{v}^{s}=0
$$

For fluid phase:

Equation (42) becomes,

$$
\frac{\partial^{2} \widetilde{C}_{2}}{\partial y^{2}}+\widetilde{C_{2}}\left(c_{3} a_{3} U_{B}^{f}+c_{1} a_{4}-\alpha^{2}\right)-c_{3} \frac{\partial C_{B 2}}{\partial y} \widetilde{v}^{f}=0
$$

Using (52) and (69), the above equation becomes,

$$
\frac{\partial^{2} \widetilde{C}_{2}}{\partial y^{2}}+\widetilde{C_{2}}\left(A_{18}+s_{21} \cosh [M y]+s_{22} \sinh [M y]-s_{23}+s_{24} e^{-\alpha_{c} y}\right)-c_{3} \widetilde{v}^{f}=0
$$

These perturbed equations (71) and (73) are solved numerically subject to the boundary conditions prescribed in (47) to (50). Graphs are plotted for the concentration without the effects of chemical reaction $\left(C_{2}\right)$, for both solid and fluid phase using MATHEMATICA.

\section{Results and Discussion}

The velocity directions are exposed to get a better perception of the flow. The velocity profile for aerosols and atmospheric fluid for parameters including Hartmann number $(M)$, electric number $(W e)$, and porous parameter $(\sigma)$ are displayed in Figures 2 and 3. Figure 2 depicts that increasing electric number and porous parameter, the velocity of aerosol increases respectively. But results vary when increasing the values of Hartmann number, where aerosol velocity decreases. Figure $3 i l l u s t r a t e s$ the same result as in Figure 2. Figure 3shows that increasing electric number and porous parameter, velocity of atmospheric fluid increases, but by increasing Hartmann number fluid velocity decreases.

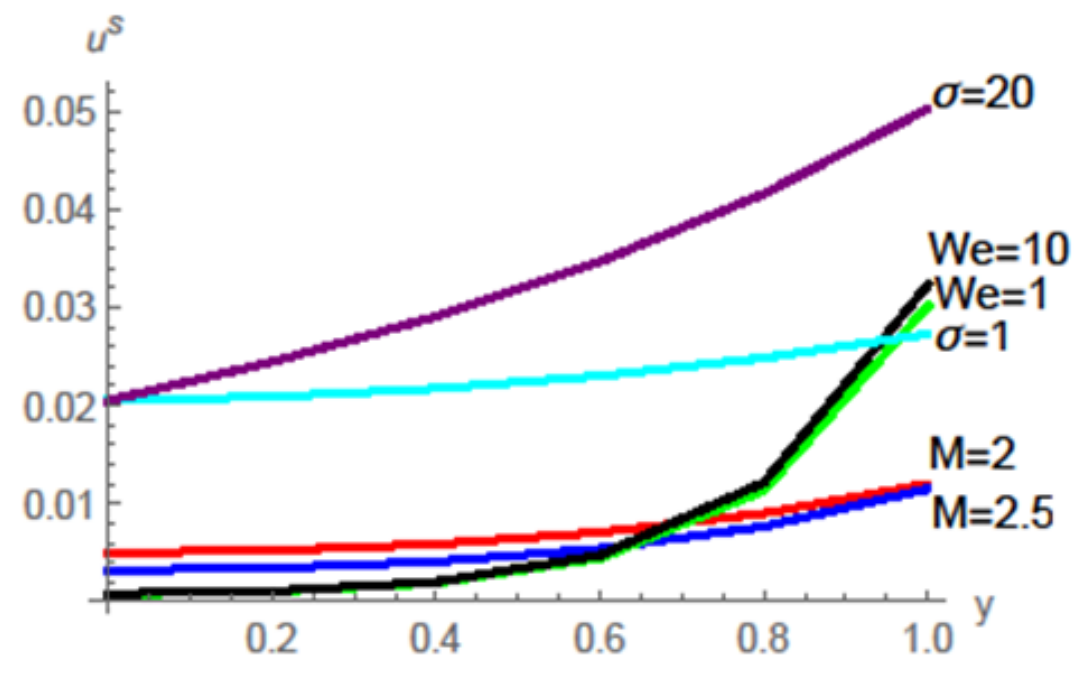

Fig 2. Velocity profile of aerosols

Concentration profiles for different values of parameters are discussed as two cases in the proceeding sub-sections. 


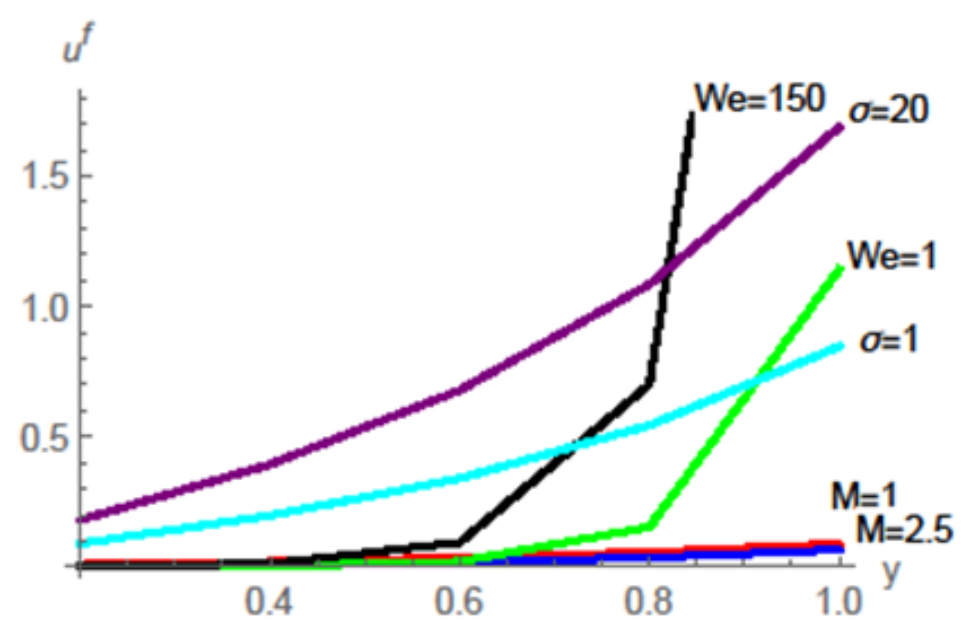

Fig 3. Plots of atmospheric fluid velocity

\subsection{Concentration of aerosol mixture in the presence of chemical reaction (when $\beta \neq 0$ ):}

The concentration of aerosols and atmospheric fluid in the presence of chemical reaction by varying some parameters are illustrated in Figure 4 to Figure 7.

Figure 4 depicts the results of aerosol concentration for some values of reaction rate parameter and Hartmann number. It reveals that increasing the rate of the reaction, aerosol concentration increases but when maximizing Hartmann number the concentration of aerosols decreases. Figure 5 represents the plots of aerosol concentration for some values of an electric and porous parameter. It reveals that increasing both electric number and porous parameter, the concentration of aerosol decreases.

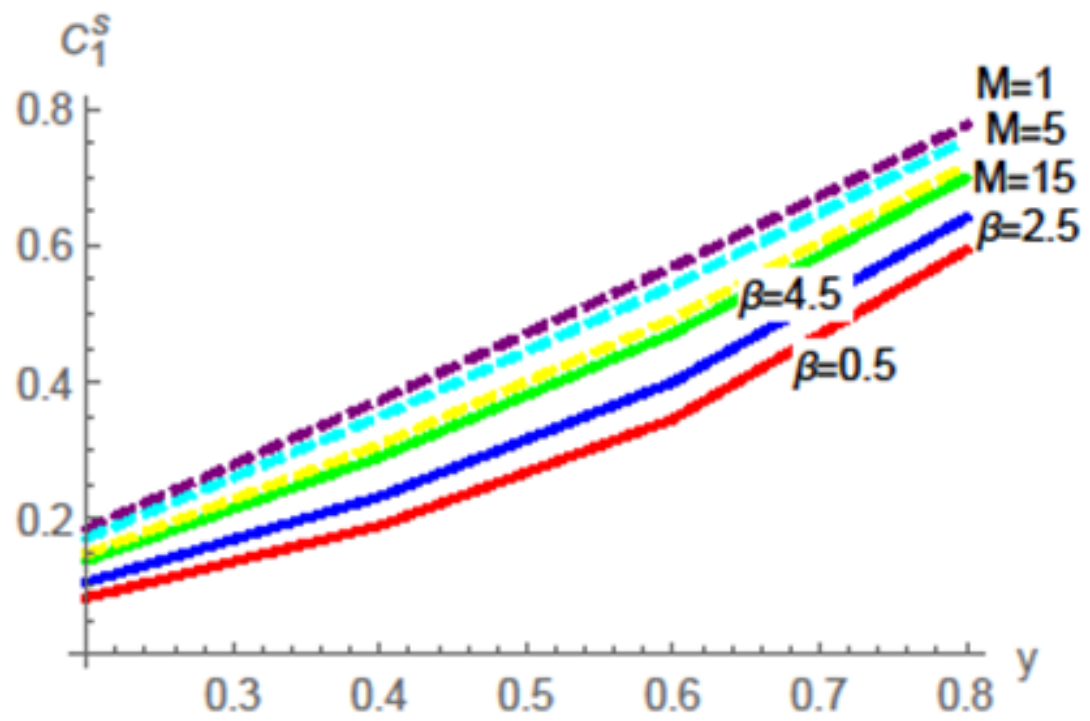

Fig 4. Concentration of aerosols for different values of reaction rate and parameter andHartmann number 


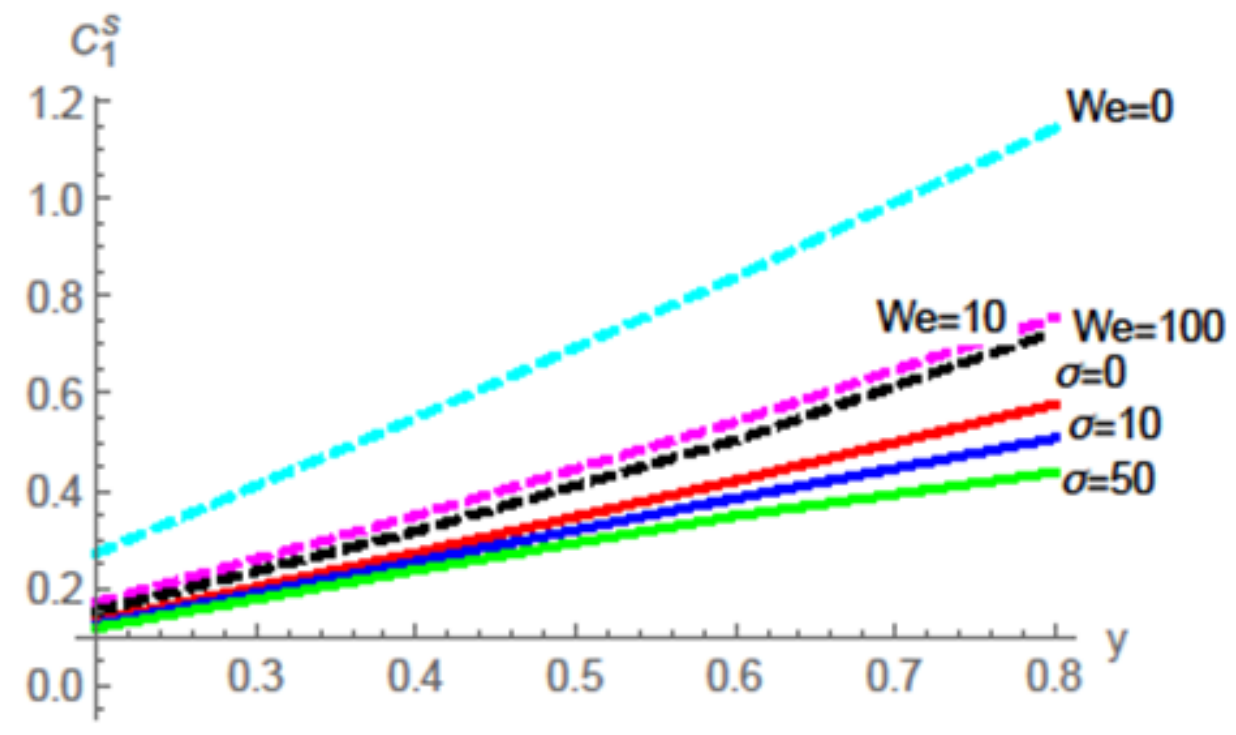

Fig 5. Concentration of aerosols for various values of electric number and porous parameter

Figure 6 illustrates the plots of fluid concentration for different values of an electric number and porous parameter. It gives a clear picture that increasing both electric number and porous parameter, fluid concentration decreases. The results of atmospheric fluid concentration for various values of reaction rate parameter and Hartmann number are presented in Figure 7. It reveals that increasing both reaction rate parameter and Hartmann number, the concentration of fluid decreases.

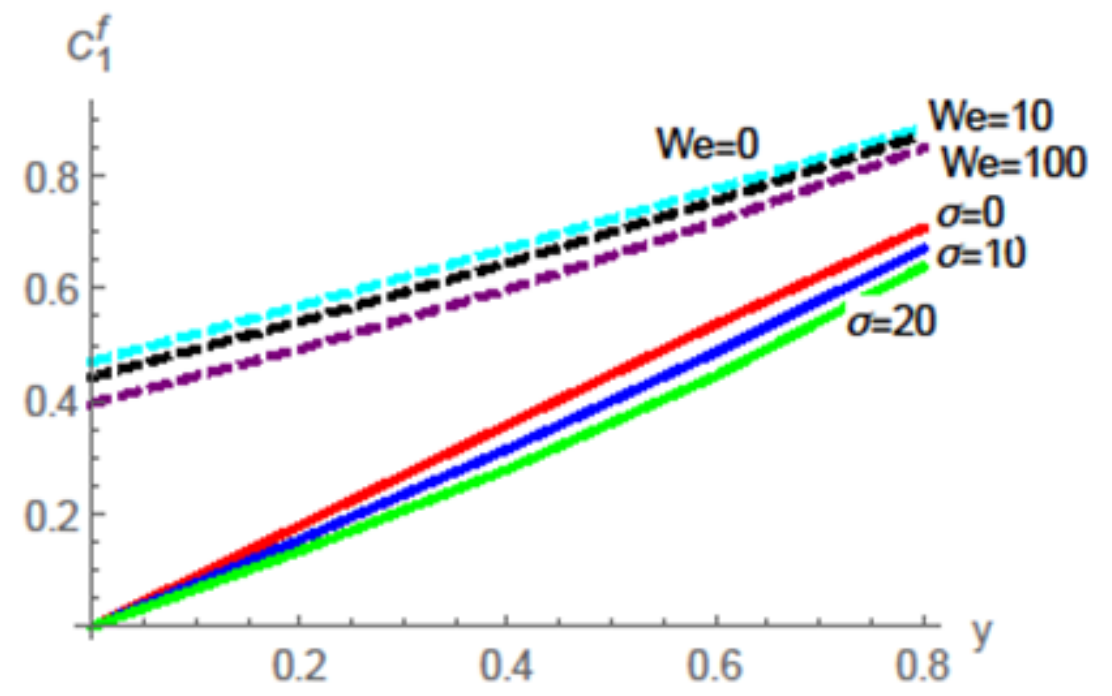

Fig 6. Concentration of atmospheric fluid for some values of electric number and porous parameter 


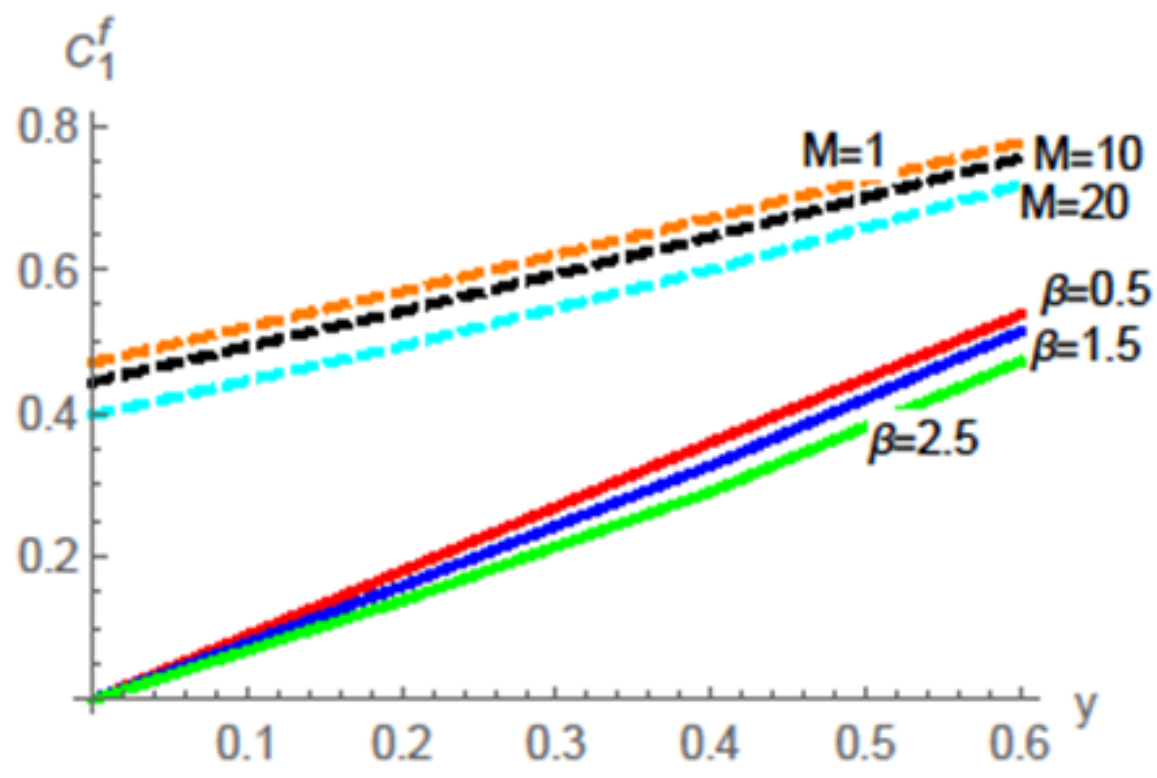

Fig 7. Concentration of atmospheric fluid for various values of reaction rate parameter and Hartmann number

\subsection{Case(2): Concentration of aerosol mixture in the absence of chemical reaction (when $\beta=0$ ):}

The concentration of aerosols and atmospheric fluid without the effect of a chemical reaction by varying some parameters are depicted in Figures 8 and 9.

Figure 8 shows the plots of aerosol concentration for different values of electric and Hartmann number. It is noted from the figure that while increasing electric number aerosol concentration maximizes. Also, increasing Hartmann number, the concentration of aerosol declines. The results of fluid concentration for various values of electric and Hartmann number are presented in Figure 9. It is observed that increasing both Hartmann number and electric number, concentration of atmospheric fluid without chemical reaction reduces.

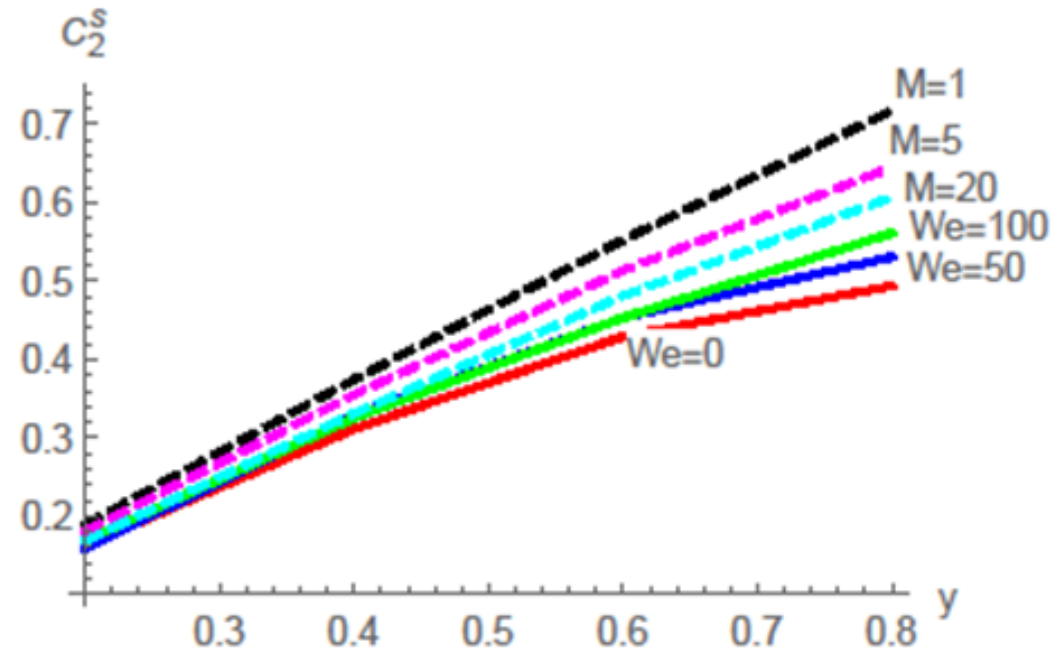

Fig 8. Concentration of aerosol for different values of Hartmann number (when $\beta=0$ ) 


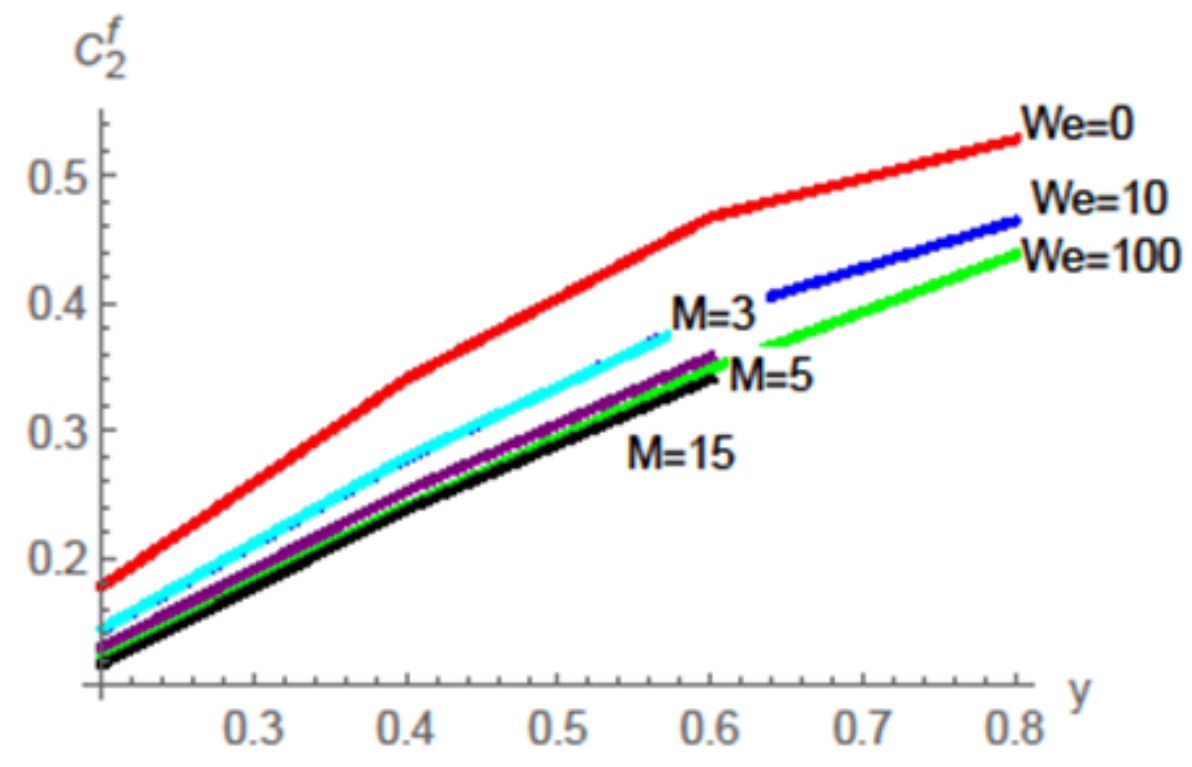

Fig 9. Concentration of atmospheric fluid for some values of Hartmann number and electric number (when $\beta=0$ )

\subsection{Discussion}

In the presence of chemical reaction,

- The concentration of aerosols in the aerosol mixture is reduced by enhancing electric, magnetic and porous effects.

- The concentration of atmospheric fluid in the aerosol mixture is reduced by increasing the rate of the reaction, and by improving electric, magnetic and porous effects.

In the absence of chemical reaction,

- The concentration of aerosols in the aerosol mixture is reduced by enhancing magnetic field effects.

- The concentration of atmospheric fluid in the aerosol mixture is reduced by increasing electric, magnetic field effects.

\section{Conclusion}

The concentration of the aerosol mixture is evaluated both in the presence and absence of homogeneous first-order chemical reaction under the presence of electric and magnetic field. The concentration of aerosols in the mixture when chemically reactive, reduces when enhancing the electric, magnetic, and porous effects. The same results hold for the concentration of atmospheric fluid in the presence of chemical reaction. Atmospheric fluid concentration in the mixture reduces while increasing the rate of the reaction, electric, magnetic and porous effects. In the absence of chemical reaction, aerosol concentration reduces when improving the magnetic field and fluid concentration reduces when maximizing the effects of both electric and magnetic fields. Hence it is concluded that both in the presence and absence of chemical reaction concentration of the mixture is minimized when electric, magnetic and porous effects are enhanced.

\section{References}

1) Barry SI, Parkerf KH, Aldis GK. Fluid flow over a thin deformable porous layer. ZAMP Zeitschrift fr angewandte Mathematik und Physik. 1991;42(5):633648. Available from: https://dx.doi.org/10.1007/bf00944763.

2) Bowen RM. Incompressible Porous Media Models by Use of the Theory of Mixtures. International Journal of Engineering Science. 1980;18(9):1129-1148. Available from: https://doi.org/10.1016/0020-7225(80)90114-7.

3) Lopatnikov SL, Gillespie JW. Poroelasticity-I: Governing Equations of the Mechanics of Fluid-Saturated Porous Materials. Transport in Porous Media. 2010;84:471-492. Available from: https://dx.doi.org/10.1007/s11242-009-9515-x.

4) Anand S, Mayya YS, Yu M, Seipenbusch M, Kasper G. A numerical study of coagulation of nanoparticle aerosols injected continuously into a large, well stirred chamber. Journal of Aerosol Science. 2012;52(52):18-32. Available from: https://dx.doi.org/10.1016/j.jaerosci.2012.04.010. 
5) Maso D, Kulmala MM, Lehtinen KEJ, kela"JMM, Aalto P, Dowd C. Condensation and Coagulation Sinks and Formation of Nucleation Mode Particles in Coastal and Boreal Forest Boundary Layers. Journal of Geophysical Research Atmospheres. 2002;107(D19):1-10. doi:10.1029/2001JD001053.

6) jun Gan F, zhong Lin J, zhou Yu M. Particle Size Distribution in a Planar Jet Flow Undergoing Shear-Induced Coagulation and Breakage. Journal of Hydrodynamics. 2010;22(4):445-455. Available from: https://dx.doi.org/10.1016/s1001-6058(09)60076-7.

7) Keller A, Siegmann HC. The role of condensation and coagulation in aerosol monitoring. Journal of Exposure Science \& Environmental Epidemiology. 2001;11(6):441-448. Available from: https://dx.doi.org/10.1038/sj.jea.7500187.

8) Seipenbusch M, Binder A, Kasper G. Temporal Evolution of Nanoparticle Aerosols in Workplace Exposure. Annals of Occupational Hygiene. 2008;52(8):707-723.

9) Dettman JW. Perturbation techniques in related differential equations. Journal of Differential Equations. 1973;14(3):547-558. Available from: https: //dx.doi.org/10.1016/0022-0396(73)90066-1.

10) Rudraiah N, Devaraju N, Ng CO. Electrohydrodynamic Dispersion of Deformable Aerosols in the Presence of an Electric Field and Chemical Reaction Using Taylor Dispersion Model. Journal of Hydrodynamics. 2011;23(2):247-257. Available from: https://dx.doi.org/10.1016/s1001-6058(10)60110-2.

11) P MP, Ratchagar NP. Coagulation and Condensation of Aerosols in Atmospheric Dispersion Model. The Journal of Computational Multiphase Flows. 2013;5(2):115-138. Available from: https://dx.doi.org/10.1260/1757-482x.5.2.115.

12) Meenapriya P. Effect of Magnetic Field on Scattering of Dust Particles with Chemical Reaction in the Atmosphere". Int J Mat Sci. 2019;18(1-2):145-154.

13) Meenapriya P, Maheswari KU, Ratchagar NP. Effects of Concentration of Air Pollutants in the Channel Bounded by Porous Beds. Advances in Mathematics: Scientific Journal. 2020;9(4):1699-1711. Available from: https://dx.doi.org/10.37418/amsj.9.4.26.

14) Dhange M, Sankad G. Peristaltic Pumping and Dispersion of a MHD Couple Stress Fluid with Chemical Reaction and Wall Effects. Journal of Advances in Mathematics and Computer Science. 2017;24(3):1-12. Available from: https://dx.doi.org/10.9734/jamcs/2017/36131.

15) Varaksin AY. Two-Phase Flows with Solid Particles, Droplets, and Bubbles: Problems and Research Results (Review). High Temperature. 2020;58:595-614. Available from: https://dx.doi.org/10.1134/s0018151x20040161.

16) Beavers GS, Joseph DD. Boundary conditions at a naturally permeable wall. Journal of Fluid Mechanics. 1967;30(1):197-207. Available from: https://doi.org/10.1017/S0022112067001375. 